Cinémas

Revue d'études cinématographiques

Journal of Film Studies

\title{
Palomar : intermédialité et archéologie de la vision
}

\section{Antonio Costa}

Volume 10, numéro 2-3, printemps 2000

Cinéma et intermédialité

URI : https://id.erudit.org/iderudit/024821ar

DOI : https://doi.org/10.7202/024821ar

Aller au sommaire du numéro

Éditeur(s)

Cinémas

ISSN

1181-6945 (imprimé)

1705-6500 (numérique)

Découvrir la revue

Citer cet article

Costa, A. (2000). Palomar : intermédialité et archéologie de la vision. Cinémas, 10(2-3), 169-184. https://doi.org/10.7202/024821ar

\section{Résumé de l'article}

Se situant dans la discussion des implications théoriques et méthodologiques des suggestions de Gérard Genette et d'Umberto Eco, l'article se propose d'étudier les interactions entre médias dans le siècle du cinéma. En particulier, on examine l'interaction entre le modèle cinématographique et l'écriture littéraire par l'analyse d'un roman d'Italo Calvino, Palornar; on convoque également d'autres textes, aussi bien de l'écrivain italien que d'autres auteurs, tous textes qui ont pour objet l'expérience visuelle, le regard et la représentation du point de vue. On arrive ainsi à tracer une sorte d'archéologie de la vision, avec des références souterraines aux dispositifs qui ont préparé l'avènement du cinéma (notamment la chronophotographie de Marey et le revolver astronomique de Jannsen). 


\section{Palomar: \\ intermédialité \\ et archéologie de la vision}

\section{Antonio Costa}

\section{RÉSUMÉ}

Se situant dans la discussion des implications théoriques et: méthodologiques des suggestions de Gérard Genette et d'Umberto Eco, l'article se propose d'étudier les interactions entre médias dans le siècle du cinéma. En particulier, on examine l'interaction entre le modèle cinématographique et l'écriture littéraire par l'analyse d'un roman d'Italo Calvino, Palomar; on convoque également d'autres textes, aussi bien de l'écrivain italien que d'autres auteurs, tous textes qui ont pour objet l'expérience visuelle, le regard et la représentation du point de vue. On arrive ainsi à tracer une sorte d'archéologie de la vision, avec des références souterraines aux dispositifs qui ont préparé l'avènement du cinéma (notamment la chronophotographie de Marey et le revolver astronomique de Jannsen).

\section{ABSTRACT}

Through a discussion of the theoretical and methodological implications of the propositions of Gérard Genette and Umberto Eco, this study proposes to correlate the interaction between medias in the century of cinema. In particular, the interaction between the cinematographic model and literary writing will be examined through the analysis of a novel by Italo Calvino (Palomar) and other nearly contemporary texts by this Italian author and others, whose texts concentrate on the visual experience, the gaze, and the representation of a point of view. The object of this essay is to trace a 
sort of archeology of vision using references that underlie the devices that paved the way for the coming of cinema (Marey's chronophotography, Jannsen's astronomic revolver, etc.).

\section{L'effet rebound}

Gérard Genette écrit dans son Nouveau Discours du récit, texte où il s'amuse à déplaire aux partisans les plus zélés de la narratologie littéraire et cinématographique (tout en ayant été le théoricien lucide de l'une et, peut-être, l'inspirateur involontaire de l'autre) : "À la différence du cinéaste, le romancier n'est pas obligé de mettre sa caméra quelque part: il n'a pas de caméra. " Mais, juste après, il se repent de cette affirmation radicale et ajoute en note: "Il est vrai qu'il peut aujourd'hui, " effet rebound" d'un médium sur l'autre, feindre d'en avoir une" (p. 49).

Il y a eu en premier lieu un effet rebound de la littérature sur le cinéma. Depuis sa naissance, le cinéma a systématiquement pillé la littérature, tirant d'elle les histoires à raconter et transposant en images, souvent pour un public analphabète, les classiques et les livres à succès. Mais il a surtout cherché à incorporer le dispositif de la narration littéraire. Dans le cinéma muet, à travers les intertitres, l'écriture se glisse dans la continuité de l'image. En outre, la métaphore du livre feuilleté vient renforcer maintes fois le déroulement de la narration; c'est le cas, entre autres de Blud of Satan Dagbog (Feuillets arrachés au livre de Satan, 1921) par Carl Th. Dreyer, auteur qui dans sa production muette a su employer la parole au-delà de sa fonction d'illustration et d'explication, en la transformant en un élément qui constitue le tissu visuel et symbolique du film. Mais avec l'avènement du cinéma parlant, il devenait possible de rendre pleinement la force de suggestion de la voix en recourant au narrateur hors cadre. C'est même par l'emploi d'une propriété du parlant (et de la radio ${ }^{1}$ ) que le cinéma a pu matérialiser ce que, dans le roman, on désignera métaphoriquement par "voix narratrice" et "narrateur-je». L'effet rebound de la littérature sur le cinéma concerne aussi, bien évidemment, la critique cinématographique, qui a transposé d'un champ à l'autre les principales 
catégories critiques et qui a adapté au cinéma, de façon plus ou moins pertinente, les modèles interprétatifs de la littérature.

Si on effectue le parcours dans l'autre sens, celui de l'effet rebound du cinéma sur la littérature, on constate que les modèles visuels ont souvent été incorporés à la littérature du siècle dernier, de la littérature américaine des années trente et quarante à l'«école du regard»des années soixante. Il a toutefois fallu les contributions plutôt récentes de la narratologie pour souligner le jeu des influences mutuelles, et ce, à partir des notions de focalisation, de point de vue, d'observateur, etc. François Jost, auteur d'une recherche complexe sur la typologie narrative à michemin entre le roman et le cinéma, a explicité son ambition de parvenir à une "narratologie comparée" (p. 9). De son côté, Genette a rappelé que la narratologie moderne, qui s'est surtout développée en France, d'où elle a amplement conditionné la recherche internationale, a eu son point de départ dans une œuvre souvent injustement oubliée, L'Âge du roman américain de Claude-Edmonde Magny ${ }^{2}$. Il s'agit d'une réflexion lucide sur les conditionnements mutuels du cinéma et de la littérature à l'époque de Faulkner, Dos Passos et Hemingway, mais aussi de Hawks, Welles et Huston de même que, en Europe, de Camus, Sartre et Malraux. La problématique soulevée par le livre de Magny a eu des échos dans la critique cinématographique, par exemple quand Rohmer, à propos de la relation entre la littérature et le cinéma américains au début des années cinquante, écrit que finalement la littérature rend au cinéma ce que le cinéma lui avait prêté: "[...] le goût pour l'ellipse, le trait précis, le visuel" (p. 14). François Jost affirme que l'application au cinéma de la problématique de la focalisation est pertinente, et même, qu'elle trouve sa raison d'être et son fondement dans le cinéma. Affirmation paradoxale? Non, si l'on pense que l'emploi (métaphorique) du mot focalisation dérive de l'optique. En paraphrasant Rohmer, on pourrait dire que la narratolgie rend au cinéma ce que le cinéma lui avait prêté.

S'agirait-il alors d'un simple emprunt linguistique? Il faut penser qu'il y a d'autres enjeux. McLuhan nous l'a appris, les médias en tant que prolongements de nos sens, changent nos relations avec le monde environnant, et donc, notre façon de lui 
correspondre et de le représenter. Comme Max Milner l'a démontré, les découvertes scientifiques et les développements de la technologie optique sont étroitement liés aux configurations du fantastique dans la littérature du XIX siècle. Mais l'idée même $\mathrm{du}$ fantastique et les techniques de sa représentation littéraire découlent d'un élargissement de l'expérience perceptive, rendu possible grâce aux nouveaux instruments et aux différentes applications de l'optique: les microscopes, les microscopes solaires et les télescopes dans le domaine scientifique, les fantasmagories de Robertson et ce qui s'ensuit dans le domaine du spectacle. Ce fort conditionnement de l'imaginaire visuel par l'imaginaire technologique se manifeste d'autant plus au $\mathrm{XX}^{\mathrm{e}}$ siècle, alors que l'imaginaire cinématographique - résultat et point d'agrégation de nombreuses technologies - joue un rôle décisif dans la construction de notre imaginaire visuel qui, comme l'a déjà dit Italo Calvino dans une de ses Leçons américaines, est l'une des composantes essentielles de l'imaginaire littéraire (1988, p. 81-98). Calvino confirme ainsi, bien qu'indirectement, la pertinence et l'efficacité de la relation supposée par Jost entre le cinéma et la littérature pour dégager des catégories narratologiques significatives.

Il ne sera pas nécessaire ici d'entrer dans le détail des travaux de Genette et de Jost. Certes, il serait facile de soulever des questions de méthode par rapport à des passages désinvoltes du plan métaphorique au littéral, et vice versa, qui caractérisent l'emploi de catégories comme la focalisation, le point de vue, le point d'écoute, etc., dans l'élan d'une fureur analytique qui, par exemple, conduit Jost à décomposer la notion de focalisation de Genette en «ocularisation" et "auricularisation" (p. 37-59). Disons simplement que l'ancienne catégorie de la focalisation se fondait sur des références équilibrées et apaisantes à l'optique, références faites pour rassurer le sens commun; tandis que l'oculaire et l' "auriculaire" de Jost, produits de l'époque des cyborgs et de l'hyperréalisme, évoquent d'inquiétantes tables d'anatomie, des enchevêtrements de fils, des opérations chirurgicales et des organes artificiels. Finalement, les chercheurs sont possédés par le même imaginaire visuel que celui des peintres, des cinéastes et des romanciers. 
Cette prolifération terminologique, tous ces petits monstres lexicaux qui se pressent autour de nous, rappellent la tératologie de Luigi Serafini où, selon la fine analyse de Calvino, "[...] l'anatomique et le mécanique échangent leur morphologie" (1984, p. 150) ${ }^{3}$. Il ne s'agit pas d'un jugement de valeur. Les monstres, et avec eux les métamorphoses et les métaphores, naissent - Calvino nous le rappelle - « [...] dans la contiguïté et la perméabilité de tout territoire de l'existence» (p. 149). Le mélange entre l'univers de l'écriture et celui de la vision (les dispositifs techniques, les images reproduites, les images dessinées), le résultat de cette contiguiité, de cette perméabilité dont nous parle Calvino à propos de Serafini, nous renvoie avec insistance à Palomar.

\section{Palomar? Qui?}

Qui est ce monsieur Palomar, sinon un petit "monstre" comme ceux de la tératologie du peintre et dessinateur Luigi Serafini? Comme les monstres de Serafini, avec lesquels il partage les traits nets mais schématiques des figures des bandes dessinées, monsieur Palomar a un corps qui semble ne pas se terminer par la tête, mais par un étrange dispositif optico-mécanique ou électronique. Une caméra, peut-être, ou une caméra vidéo. $\mathrm{Ou}$ encore un télescope, ou un capteur à fibres optiques. Plus simplement, s'agirait-il d'une miniaturisation du miroir du télescope de ce mont Palomar dont il est l'homonyme? Dans ce jeu entre le subjectif et l'instrumental, et même dans la métaphore constitutive du personnage, on recherche une sorte d'objectif corrélatif d'une vue sans sujet, ou encore de l'anéantissement de la subjectivité en une fonction perceptive: «[...] le moi ne serait-il rien d'autre que la fenêtre à travers laquelle le monde regarde le monde?" $\left(1985\right.$, p. 112) ${ }^{4}$.

Dans les minutieuses descriptions de Calvino, le regard de monsieur Palomar semble parfois bouger avec une régularité chronométrique, comme le regard de la caméra dans les panoramiques guidés par la perfection géométrique des paliers à billes, et dans les évolutions de la steady cam associant d'abstraites régularités à une légèreté liquide. Plus souvent, les dispositifs évoqués par le regard de monsieur Palomar ont quelque chose 
d'ancien. Des stades primitifs de l'évolution technologique semblent ressortir dans les comportements et les obsessions de ce produit terminal de l'âge de la vue mécanique, et des modèles précédents de l'évolution biologique se manifestent dans des comportements humains, selon les théories d'Henri Laborit, qui ont inspiré Mon oncle d'Amérique (Alain Resnais, 1980). Chapitre après chapitre, on pourrait aussi dire séquence après séquence, monsieur Palomar semble être destiné à parcourir de nouveau les lieux, les stades de cette obsession visuelle qui soude dans une continuité floue et ambiguë la fin du XIX siècle à celle du $X^{e}$ siècle. Peut-être, parce qu'il y a de la part de Palomar le choix de se "construire un rapport au monde limité à l'observation des formes visibles" $(1985, \text { p. } 56)^{5}$, son itinéraire est-il destiné à passer maintes fois par les lieux figés de cette prééminence du visible qui a dominé l'imaginaire scientifique pendant un siècle et qui se conclut avec l'invention du cinéma. Ainsi, le regard de Palomar semble parfois fonctionner comme le fusil chronophotographique d'Étienne J. Marey; et d'autres fois, comme le revolver photographique de Pierre-Jules C. Janssen.

Monsieur Palomar à la plage, devant le problème de la «lecture d'une vague", semble revivre toutes les obsessions de la recherche optique-physiologique du professeur Marey, dont le premier film chronophotographique s'intitule justement $L a$ Vague (1890). Grâce à ses recherches sur l'impression photographique du mouvement, Marey figure parmi les pionniers du cinéma. Ces recherches l'ont amené à la mise au point d'un système d'enregistrement (le chronophotographe) et à l'ébauche d'un système de projection des images en mouvement. Marey était un médecin, un physiologiste: il se proposait d'analyser le mouvement, de traduire en courbes et en diagrammes les aspects que l'œil humain, trop plongé dans le tourbillon de la vie, ne pouvait saisir et "fixer". S'il a abandonné ses recherches sur le projecteur chronophotographique, c'est qu'il ne voyait aucun intérêt à projeter sur l'écran "la vie telle qu'elle est ». Son idéal a été atteint quand "[...] les images animées se sont immobilisées en des figures géométriques: l'illusion des sens s'est évanouie, mais elle a fait place à la satisfaction de l'esprit " (Deslandes, p. 144). 
Monsieur Palomar à la plage rappelle une gravure très connue des historiens du cinéma des origines, qui montre Marey au bord de la mer avec son fusil chronophotographique, le dispositif avec lequel il chercha à fixer dans des tracés très rigoureux la palpitation indéfinie de l'univers, à retenir, à garder la trace " [...] des mouvements les plus subtils, les plus fugitifs de la vie" (Collet, p. 41). Certes, avec cette idée de la maîtrise absolue du visible, Marey est complètement plongé dans l'idéologie de la souveraineté du regard (l'œil absolu qui fait de la vie un cadavre) dont parle Michel Foucault dans son Archéologie du regard médical (1963). Comme Marey, dont il semble revivre les obsessions, monsieur Palomar scrute dans le ciel le vol des oiseaux en cherchant une méthode pour " [...] fixer dans les moindres détails le peu qu'il arrive à voir» $\left(1985\right.$, p. 64) ${ }^{6}$. En effet, cette idée de fixer tout ce qui est fuyant, merveilleusement exprimée dans le chapitre "L'invasion des volées", présente des analogies extraordinaires avec les écrits de Marey: franchir les limites de la vision trop impliquée dans le tourbillon de la vie, compromise dans le mouvement convulsif du vol, saisie par les fantômes du désir; trouver l'ordre, la symétrie, la régularité mesurable, posséder ce qui nous échappe par le signe, la trace, le nom.

Monsieur Palomar n'ignore pas que cette obsession de figer les choses, de les nommer, de les classer, a pour effet de les pétrifier: "[...] son regard transforme chaque plat en un document de l'histoire de la civilisation, en un objet de musée" (p. 73) ${ }^{7}$, lit-on dans le chapitre "Palomar fait le marché». Ce qui nous frappe pourtant, parmi les résultats des exercices chronophotographiques de Marey, c'est, sans aucun doute, la sculpture en bronze des différentes étapes du vol d'un goéland, extraordinaire aussi du point de vue esthétique pour ses anticipations du futurisme et du cubisme ${ }^{8}$. L'impression de "muséification" du frémissement à la fois régulier et haletant de la vie est très forte: le résultat est, justement, monstrueux, étant donné qu'il se produit dans la contiguïté du mobile et de l'immobile, du flottant et du rigide, de la répétition et de la différence, de l'unité et de la pluralité. À cet égard, il n'est pas anodin que Calvino ait placé en introduction à Collezioni di sabbia, qu'il faudrait lire en parallèle avec Palomar', un article de 1974 
qui donnera, plus tard, par un jeu de mise en abyme, son titre au livre et dans lequel on lit ceci :

Et pourtant, celle qui a eu la constance de poursuivre des années durant cette collection savait ce qu'elle faisait, savait où elle voulait arriver : peut-être justement à éloigner d'elle le vacarme des sensations déformantes et agressives, le vent confus du vécu, et à avoir pour elle enfin la substance sableuse de toutes choses, à toucher la structure de silice de l'existence (1986, p. 16-17) ${ }^{10}$.

Jean Collet, en commentant les thèmes de la recherche de Marey, rappelle que dans Le Septième Sceau (Bergman, 1956), il y a un jongleur qui essaie d'apprendre à son fils un numéro extraordinaire consistant à immobiliser une balle au beau milieu de sa trajectoire. Il associe cet exercice à l'image, au début du film, de l'aigle planant et figé en vol que Bergman tire de l'Apocalypse. Collet écrit :

[...] cet oiseau pétrifié en vol nous livre la signification du texte johannique et du film à la fois: il marque l'instant paradoxal où le temps cesse d'exister, instant au seuil de la mort $[\ldots]$ où l'homme doit avoir la révélation ultime, la connaissance suprême, le secret de la vie (p. 41).

Monsieur Palomar scrute l'univers dans l'espoir de pouvoir accéder, pourrait-on dire en paraphrasant Blanchot, à une "deuxième vue». Personnage tragique et burlesque à la fois - il est suspendu entre l'intériorisation du temps vécu et l'objectivation de comportements machinaux et répétitifs - , Palomar adapte les modalités de son regard à l'aptitude analytique des différents outils de "l'histoire de la vision». Ainsi est-il toujours sur le point de nous rendre, tout comme le fusil chronophotographique de Marey, le profil pétrifié de l'univers, la sublimation abstraite (ou la dégradation mécanique?) du frémissement vital des choses. Comme Janssen avec son revolver astronomique, $\mathrm{Pa}$ lomar interroge les traces visibles de l'évolution des astres dans l'univers, cherchant les correspondances entre l'étroite perspective terrestre et l'immensité des espaces célestes. Mais qu'arrivera-t-il, quand «il pointera son télescope sur les orbites 
tracées par le cours de sa vie, plutôt que sur celles des constellations" ? :

Que verra-t-il? Son monde intérieur lui apparaîtra comme l'immense rotation d'une spirale lumineuse? Verra-t-il les étoiles et les planètes naviguer en silence sur les paraboles et les ellipses qui déterminent son caractère et son destin? Contemplera-t-il une sphère à la circonférence infinie qui a pour centre le moi et qui a le centre en tous ses points $\left(1985\right.$, p. 117) ${ }^{11}$ ?

\section{Le regard et le tressaillement}

Palomar semble destiné à poursuivre sans cesse sa recherche et à enrichir avec une précision maniaque son encyclopédie des mouvements, sa collection de perceptions, son catalogue de sensations, son étalage d'événements. Ce qu'il cherche et qui lui échappe toujours est une chose rare et précieuse: elle est révélée dans le chapitre "Le monde regarde le monde". Monsieur Palomar cherche "[...] une de ces heureuses coïncidences dans lesquelles le monde veut regarder et être regardé en même temps " $\left(1985\right.$, p. 113) ${ }^{12}$. Il n'est pas difficile de voir là une référence à la théorie phénoménologique de la vision énoncée par MerleauPonty ou, dans le domaine du cinéma, par André Bazin (qui s'est inspiré de cette théorie) ${ }^{13}$. L'une de ces coïncidences se trouve décrite dans le deuxième chapitre, "Le sein nu». Elle est marquée par un "écart », par une "différente consistance de la vision»:

Maintenant, en parcourant la plage du regard avec une objectivité impartiale, il fait en sorte qu'à peine la poitrine de la femme entre dans son champ visuel. On y remarque une discontinuité, un écart, presque un éclair. Le regard avance jusqu'à effleurer la peau tendue, se retire, comme s'il appréciait, avec un léger tressaillement, la différente consistance de la vision et la valeur particulière qu'elle assume, et pour un instant il se suspend en l'air, décrivant une courbe qui, de façon évasive et à la fois protectrice, accompagne le relief du sein à distance, pour reprendre ensuite son cours comme si de rien n'était (p. 17) ${ }^{14}$.

Greimas, qui a consacré à ce chapitre une analyse exemplaire (p. 23-33), a certes raison quand il rappelle "la conception 
d'Husserl de la perception " et souligne que l'objet esthétique (dans ce cas-là la poitrine de la femme) ne se constitue définitivement qu'à travers la production d'une discontinuité sur le continu de l'espace visuel (p. 28) ${ }^{15}$. Mais ce que Greimas ne relève pas, c'est que, dans tout le chapitre, l'analyse des mouvements du regard de Palomar, le passage du plan général au détail, l'idée même de "discontinuité » - terme que le critique emprunte directement à Calvino - entre les différents moments de la vision, bref, tous les aspects qui caractérisent mieux l'imaginaire visuel inspirant ce texte renvoient avec insistance à la technique de tournage cinématographique (sans compter la relation de voyeurisme qui s'établit entre le sujet et l'objet, un autre renvoi à une situation typiquement cinématographique).

Il s'agit d'un exemple splendide d'effet rebound: CalvinoPalomar, comme le romancier dont parle Genette, semble feindre d'avoir une caméra; il semble même regarder la réalité à travers l'objectif. L'idée même de discontinuité, d'écart, de consistance différente de la vision est en parfaite correspondance avec la définition, autant intuitive qu'analytique, de "subjectif». Il s'agit ainsi d'une sorte de rencontre entre l'objectivité impartiale du regard de monsieur Palomar (qui s'attribue une qualité typique du regard cinématographique) et la subjectivité du désir menant le mouvement même du regard à produire une des configurations du "réalisme ontologique" dont Bazin parlait. Configuration qui apparaît très proche des conditions dans lesquelles "l'énigme de la vision" se réalise en tant que, d'après MerleauPonty, "[...] concordance spontanée entre l'aspect subjectif et l'aspect objectif du phénomène», et "[...] harmonie entre les projets moteurs et le monde aperçu" (Sordini, p. 179).

À la manière de Genette et d'Eco, on pourrait affirmer que Calvino maîtrise avec une efficacité enviable la caméra subjective. Dans ce cas, l'effet rebound ne fonctionne pas seulement sur le plan de l'écriture, mais aussi sur celui de la réception: une lecture de ce genre implique l'activation des soi-disant «scénarios visuels intertextuels" ou "scénarios iconiques" dont parle Umberto Eco (p. 81), proches des configurations du langage cinématographique. L'étrange configuration de ce plan subjectif particulier (lié à un mouvement, à un regard mobile, qui réitère 
son parcours jusqu'au point où il aperçoit une différence, un écart) évoque le mouvement du regard. Mouvement qui, devant une anamorphose, attend l'instant où la prise de vue exacte provoquera finalement le jaillissement de l'image cherchée, désirée, le dévoilement, le sens caché de l'enchevêtrement apparemment informe des lignes ${ }^{16}$. La "lecture visuelle du monde" de Calvino, suppose la capacité de saisir l'écart, la discontinuité, les différences sans lesquelles «[...] le regard glisse sur une surface lisse et sans prises ". Les perceptions de l'écart et de la différence, fugaces et intermittentes, sont en jeu dans l'économie du désir, parce qu'elles ouvrent le regard sur des dimensions diverses, réglées par des espaces et des temps différents: c'est ce qui lie la discontinuité au tressaillement, au frémissement.

Le mot "tressaillement" (trasalimento) n'est pas nouveau dans le lexique de Calvino: il figurait déjà dans un des chapitres «japonais" de Se una notte d'inverno un viaggiatore, où l'on traite de la perception de la discontinuité dans le champ visuel (p. 156). Ce même mot, trasalimento, apparaît aussi dans la traduction italienne d'un texte de Starobinski consacré au thème du regard et du désir où, malgré sa fonction distincte (il est attribué à la forme observée et non pas au regard), il présente des analogies singulières avec l'aptitude régissant le regard de monsieur Palomar; tant qu'il semble légitime d'en supposer la connaissance par Calvino. Dans L'CEil vivant, Starobinski rappelle qu'en français le mot regard, qui désigne la vision orientée, n'indiquait originairement pas l'action de voir, mais plutôt l'attente, la préoccupation, la garde, la sauvegarde, et il poursuit ainsi :

Regarder est un mouvement qui vise à reprendre sous garde... L'acte du regard ne s'épuise pas sur place: il comporte un élan persévérant, une reprise obstinée, comme s'il était animé par l'espoir d'accroître sa découverte et de reconquérir à nouveau ce qui lui est en train d'échapper. Ce qui m'intéresse est le destin de l'énergie impatiente qui habite le regard et qui désire autre chose que ce qui lui est donné : guettant l'immobilité dans la forme en mouvement, à l'affût du plus léger frémissement ${ }^{17}$ dans la figure au repos, demandant à rejoindre le visage derrière le masque, ou cherchant à se reprendre à la fascination vertigineuse des profondeurs pour 
retrouver, à la surface des eaux, le jeu des reflets

(p. 11-12).

Entre la pétrification et le tressaillement (frémissement), entre la vérité ultime et le vide inaugural de chaque regard, s'étendent le répertoire, l'exposition et la collection infinis.

Ce n'est pas fortuit si, dans Collezione di sabbia (un ouvrage consacré à des cartes, des musées, des expositions, des encyclopédies, des collections, des répertoires, réels ou imaginaires), la position centrale est occupée par la section "Il raggio dello sguardo" - "Le rayon du regard ${ }^{18}$ ". Celle-ci s'ouvre sur un texte à la mémoire de Roland Barthes, presque entièrement consacré à La Chambre claire, un livre sur la photographie, ou mieux, sur l'imaginaire photographique écrit par un auteur dont l'œuvre «[...] consiste à obliger l'impersonnalité du mécanisme linguistique et cognitif à tenir compte de la corporéité du sujet vivant et mortel" (1984, p. 78). Le texte conclusif consiste en une critique de L'occhio e l'idea. Fisiologia e storia della visione de Ruggero Pierantoni, un livre qui, en traçant l'histoire des mythes liés à la vision, amène Calvino à se demander s'il ne faudrait pas tirer la conclusion que l'esprit humain fonctionne seulement grâce aux mythes et que "[...] l'alternative ne réside que dans l'adoption d'un code mythique plutôt qu'un autre" (p. 126).

Si Palomar est l'histoire d'une obsession visuelle, Collezione di sabbia est en quelque sorte l'archéologie de cette obsession. "Il raggio dello sguardo " contient un passage consacré aux fouilles archéologiques de Settefinestre, près d'Orbetello. Ici, Calvino cite un néologisme, traulare (de l'anglais trowel: "truelle»), forgé par les ouvriers des fouilles. Par ce verbe, explique-t-il, on désigne l'opération de mettre à jour, de photographier et de dessiner, de figer couche après couche, fragment après fragment, les restes du passé (p. 87-93).

Si cette petite truelle introduite par les archéologues anglais peut être considérée comme "l'emblème symbolique de la nouvelle archéologie", Collezione di sabbia est une tentative de traulare les différentes couches de cet imaginaire visuel dont Palomar offre un répertoire significatif. Des automates de Neuchâtel à l'iconographie des "faits divers», des monstres en cire du doc- 
teur Spitzer aux freaks de Leslie Fiedler (et de Tod Browning), de la «lecture visuelle» de "l'empire des signes» japonais aux "récits du fantastique", Calvino visite et relit les lieux et les moments de la formation de l'imaginaire visuel à notre époque. Même si dans Palomar il n'y a pas de référence explicite au cinéma, l'effet rebound et la présence d'obsessions typiques de "l'archéologie du cinéma " font de ce texte l'un des plus significatifs pour l'étude des rapports entre la littérature et les médias à l'ère actuelle, rapports sur lesquels Calvino reviendra dans "Visibilité", la quatrième de ses Leçons américaines, non sans un accent nostalgique pour un âge archaïque des médias: "Mon expérience et ma formation initiale sont déjà celles d'un héritier de la "civilisation des images". " L'image visuelle l'emporte sur l'expression verbale, à une époque comme la nôtre "[...] où la littérature, sans plus se réclamer d'une autorité ni d'une tradition qui serait son origine et sa fin, mise sur la nouveauté, sur l'originalité, sur l'invention" (1989, p. 149-150).

Devant l'inflation des images préfabriquées, il y a, selon Calvino, deux voies possibles: la voie du postmodernisme qui consiste à «[...] recycler les images usées, en les insérant dans un nouveau contexte qui en modifie le sens ", à employer ironiquement l'imaginaire de la culture de masse ou à introduire " [...] le goût du merveilleux, hérité de la tradition littéraire, dans des mécanismes narratifs qui en renforcent le pouvoir d'étrangeté" (p. 141). L'autre voie est celle que suit Samuel Beckett, et qui consiste à " $[\ldots]$ faire le vide pour repartir à zéro", en réduisant " [...] au minimum les éléments visuels et le langage, comme dans un monde après la fin du monde» (p. 154).

Palomar se situe au carrefour de ces deux voies. Il y a un vide programmatique construit autour de ce personnage qui scrute la surface des choses, explore d'une manière obsessionnelle les détails, réduit sa relation au monde à une relation visuelle. Ainsi, il revit, comme nous avons cherché à le démontrer, une "archéologie» de la vision, une stratigraphie des « relations visuelles». Il y a quelque chose de mécanique, de non naturel dans les différentes attitudes auxquelles Palomar adapte son regard. Il s'agit pourtant de la même automaticité du grand burlesque, qui rend les héros du cinéma muet tragiques et dérisoires à la fois. C'est 
ainsi qu'à la fin, nous imaginons monsieur Palomar: avec les traits d'un héros du burlesque américain, un Buster Keaton désormais sans âge qui, comme dans Film (Beckett et Schneider, 1965), survit dans les limbes d'une après-histoire, avec la terreur que les cauchemars de son (ou de notre) histoire ne reviennent le hanter.

\author{
Texte français établi avec \\ le concours de Silvestra Mariniello
}

Université de Bologne

\title{
NOTES
}

1 Il ne faut pas oublier que le cinéma sonore doit beaucoup à la radio, tant sur le plan du développement technique qu'en ce qui concerne la thétorique des effets sonores.

2 Voici ce que Genette a écrit à propos de cet essai dans Nouveau Discours du récit (Paris: Seuil, 1983): "Cette étude aujourd'hui méconnue, et que l'on pille souvent sans le dire et parfois sans le savoir, fut à bien des égards le point de départ de la narratologie française, stimulée à travers elle par la tencontre du roman américain et de la technique cinématographique. Son oubli dans la bibliographie du Discours du récit est tout à fait caractéristique et d'autant plus injustifrable que, l'ayant lue et admirée dès sa première publication, je l'avais signalée en 1966, dans le dossier du $\mathrm{n}^{\circ} 8$ de Communications. Souvenir exposé à éclipses" (p. 44). En effet, dans Communications ( $\mathrm{n}^{\circ} 8$, 1966), Genette écrit que l'étude de Magny "a fortement contribué à reverser sur la littérature l'intérêt que la nouveauté des moyens cinématographiques avait suscité autour des problèmes de la technique narrative» (p. 166).

3 Le texte sur Luigi Serafini n'est pas compris dans l'édition française de Collezione di sabbia.

4 En italien : " [...] forse l'io non è altro che la finestra attraverso la quale il mondo guarda il mondo" (Italo Calvino, Palomar, Torino: Einaudi, 1983, p. 116).

5 En italien: "Quando Palomar s'era accorto di quanto approssimativi e votati all'errore sono i criteri di quel mondo dove credeva di trovare precisione e norma universale, era tornato lentamente a costruirsi un rapporto col mondo limitato all'osservazione delle forme visibili" (Italo Calvino, Palomar, Torino: Einaudi, 1983, p. 54; c'est moi qui souligne).

6 En italien : "[...] fissare nei minimi dettagli il poco che riesce a vedere " (Italo Calvino, Palomar, Torino: Einaudi, 1983, p. 63).

7 En italien: « [...] il suo sguardo trasforma ogni vivanda in un documento di storia della civiltà, in un oggetto da museo " (Italo Calvino, Palomar, Torino: Einaudi, 1983, p. 72).

8 Voir en particulier la Sculpture en bronze du vol de goéland (1887), conservée au musée de Beaune et reproduite dans un ouvrage de Michel Frizot intitulé La Chronophotographie (Dijon: Association des Amis de Marey, 1984, p. 39).

9 En ce qui concerne les rapports entre Palomar et Collezione di sabbia, on se reportera aux propos de Gian Carlo Ferreti dans Le capre di Bikini. Calvino giornalista e 
saggista 1945-1985 (Roma: Editori Riuniti, 1989, p. 145-153). Ferretti y montre «les très étroits liens existant entre la série jounalistique de Collezioni di sabbia et la série littéraire de Palomar».

10 En italien: «Eppure, chi ha avuto la costanza di portare avanti per anni questa raccolta sapeva quel che faceva, sapeva dove voleva arrivare, forse è proprio per allontanare da sè il frastuono delle sensazioni deformanti e aggressive, il vento confuso del vissuto, ed avere finalmente per sè la sostanza sabbiosa di turte le cose, toccare la struttura silicea dellesistenza" (Italo Calvino, Collezione di sabbia, Milano: Garzanti, 1984, p. 13).

11 En italien: "Cosa vedrà? Gli apparirà il suo mondo interiore come un calmo immenso ruotare d'una spirale luminosa? Vedrà navigare in silenzio stelle e pianeti sulle parabole e le ellissi che determinano il carattere e il destino? Contemplerà una sfera di circonferenza infinita che ha l'o per centro e il centro in ogni punto?" (Italo Calvino, Palomar, Torino: Einaudi, 1983, p. 121-122).

12 En italien: "[...] una di quelle fortunate coincidenze in cui il mondo vuole guardare ed essere guardato nel medesimo istante" (Italo Calvino, Palomar, Torino: Einaudi, 1983, p. 117).

13 À ce sujet, on consultera l'ouvrage de Dudley Andrew, André Bazin (New York: Oxford University Press, 1979, p. 229 ssq).

14 En italien: "Ora, nel far scorrere il suo sguardo sulla spiaggia con oggettività imparziale, fa in modo che appena il petto della donna entra nel suo campo visivo, si noti una discontinuità, uno scarto, quasi un guizzo. Lo sguardo avanza fino a sfiorare la pelle tesa, si ritrae, come apprezzando un lieve trasalimento la diversa consistenza della visione e lo speciale valore che essa acquista, e per un momento si tiene a mezzaria, descrivendo una curva che accompagna il rilievo del seno da una certa distanza, elusivamente ma anche protettivamente, per poi riprendere il suo corso come niente fosse" (Italo Calvino, Palomar, Torino: Einaudi, 1983, p. 56).

15 Voici ce que dit exactement Algirdas Julien Greimas: «Doté de la fonction syntaxique du sujet, construit au milieu du champ perceptif par la protensivité du regard, l'objet esthétique ne se constitue qu'en produisant de la discontinuité sur le continu de l'espace visuel" (De l'imperfection, Périgueux: Pierre Fanlac, 1987, p. 28). On pourra se reporter, en ce qui concerne les rapports entre "éros et discontinuité ", aux réflexions de Calvino dans Collection de sable (Paris: Seuil, 1984, p. 111-112), réflexions portant sur les gravures érotiques japonaises.

16 En ce qui concerne les rapports entre vue anamorphique et vue cinématographique, voir l'article d'Antonio Costa et Manlio Brusatin, "Visione" (Enciclopedia, vol. 14, Torino: Einaudi, 1981, p. 1110-1138). Sur les rapports entre travelling et anamorphose, voir l'ouvrage de Pascal Bonitzer, Décadrages. Cinéma et peinture (Paris: Éditions de l'Étoile, 1985, p. 93-95). Bonitzer écrit: "Ce que démontrent les anamorphoses, c'est que la peinture a, non moins que le cinéma et depuis longtemps, sinon depuis toujours, affaire au mouvement. Le point de vue se déplace et ce déplacement est un déplacement de sens. Au cinéma aussi, le mouvement d'appareil, les changements de plan, sont le mouvement même du sens" (p. 95).

17 Il faut remarquer que le traducteur italien du texte de Starobinski a traduit frémissement par ce trasalimento (Jean Starobinski, L'occhio vivente, Torino: Enaudi, 1975, p. 7).

18 La traduction française (partielle) ne respecte pas la structure de l'édition italienne. 


\section{OUVRAGES CITÉS}

Andrew, Dudley. André Bazin. New York: Oxford University Press, 1979.

Bonitzer, Pascal. Décadrages. Cinéma et peinture. Paris: Éditions de l'Étoile, 1985.

Calvino, Italo. Leçons américaines. Aide-mémoire pour le prochain millénaire. Paris: Gallimard, 1989.

Calvino, Italo. Lezioni americane. Sei proposte per il prossimo millennio. Milano: Garzanti, 1988.

Calvino, Italo. Collection de sable. Paris : Seuil, 1986.

Calvino, Italo. Palomar. Paris: Seuil, 1985.

Calvino, Italo. Collezione di sabbia. Milano: Garzanti, 1984.

Calvino, Italo. Palomar. Torino: Einaudi, 1983.

Calvino, Italo. Se una notte d'inverno un viaggiatore. Torino: Einaudi, 1979.

Collet, Jean. "Caméra ". Lectures du film (collectif). Paris: Albatros (1976), p. 37-45.

Costa, Antonio et Manlio Brusatin. "Visione », Enciclopedia, vol. 14. Torino: Einaudi (1981), p. 1110-1138.

Deslandes, Jacques. Histoire comparée du cinéma, tome I. Tournai : Casterman, 1966.

Eco, Umberto. Lector in fabula. Milano: Bompiani, 1979.

Ferretti, Gian Carlo. Le capre di Bikini. Calvino giornalista e saggista 1945-1985. Roma: Editori Riuniti, 1989.

Foucault, Michel. Naissance de la clinique. Une archéologie du regard médical. Paris: P.U.F., 1963.

Frizot, Michel. La Chronophotographie. Dijon : Association des Amis de Marey, 1984.

Genette, Gérard. Nouveau Discours du récit. Paris: Seuil, 1983.

Greimas, Algirdas Julien. De limperfection. Périgueux : Pierre Fanlac, 1987.

Jost, François. L'Eil-caméra. Entre film et roman. Lyon: Presses Universitaires de Lyon, 1987.

Magny, Claude-Edmonde. L’Âge du roman américain. Paris: Seuil, 1948.

Milner, Max. La Fantasmagorie. Essai sur l'optique fantastique. Paris : P.U.F., 1982.

Pierantoni, Ruggero. L'occhi e l'idea. Fisiologia e storia della visione. Torino: Boringhieri, 1982.

Rohmer, Éric. "Redécouvrir l'Amérique". Cahiers du cinéma, $n^{\circ} 54$ (1955), p. 11-16.

Sordini, Anna. "Pittura e metafisica nell'ultimo Merleau-Ponty", dans Franco Fergnani (direction), Il corpo vissuto. Milano: Il Saggiatore, 1979. 